\title{
La calidad de la democracia y sus factores determinantes. Un análisis comparado de 60 países
}

\author{
Alex Tusell Collado \\ Universitat Oberta de Catalunya \\ atusell@uoc.edu
}

Recibido: 03-07-2014

Aceptado: 27-01-2015

\section{Resumen}

Esta investigación estudia de forma comparada la calidad de la democracia en 60 países. Se identifican seis dimensiones de la calidad democrática y se obtiene, con un análisis factorial, un índice agregado. Los datos indican diferencias substanciales entre los países. Se observan disimilitudes entre las democracias del "norte" y del "sur". El análisis de regresión múltiple indica que el capital social es el factor más explicativo de estas diferencias y que la experiencia democrática también influye en la calidad de la democracia. El análisis discrepa con las tesis estructurales de la literatura en no encontrar significativos el desarrollo económico y la desigualdad. El análisis también indica que los sistemas electorales mayoritarios tienen mejor calidad democrática y que el multipartidismo no influye negativamente en la calidad de la democracia.

Palabras clave: calidad democrática, democracia, medición de la democracia, derechos, capital social, instituciones políticas. 


\title{
The Quality of Democracy and its Determinants. A Comparative Analysis of 60 Countries
}

\begin{abstract}
This paper examines, in a comparative way, the quality of democracy in 60 countries. Six aspects of democratic quality are identified and this conclusion is the result of an factorial analysis, an aggregate indicator. This shows a big contrast between the countries. There are differences between the "north" and "south" democracy. The multiple regression analysis shows that social capital is the main reason of these differences and the democratic experience also influences democracy quality. The analysis disagrees with the structural thesis of literature because it doesn't find significant the economic development and the inequality. The analysis also shows that majoritary electoral systems have got better quality of democracy and the multipartidism doesn't affect negatively the quality of democracy.
\end{abstract}

Keywords: Democratic Quality; Democracy; Measuring Democracy; Rights; Social Capital; Political Institutions.

\section{Referencia normalizada}

Tusell Collado, Alex (2015): "La calidad de la democracia y sus factores determinantes. Un análisis comparado de 60 países”, Política y Sociedad, 52 (1), pp. 179-204.

Sumario: 1. Introducción. 2. ¿Qué se entiende por calidad de la democracia? 3. Medir la calidad de la democracia. 4. Factores explicativos de la calidad de la democracia. 5. Exploración de los factores explicativos de la calidad de la democracia. 6. Conclusiones. 7. Bibliografía. 


\section{Introducción}

Con la expansión de la democracia como régimen político, ${ }^{1}$ el interés de la literatura se ha desplazado de la teoría de la democratización hacia el análisis de las características y el rendimiento de la democracia. Concretamente, uno de los temas de interés de la academia es el estudio de la calidad de la democracia, pero se trata de un tema complejo con diversas aproximaciones teóricas y empíricas.

Esta investigación pretende, en primer lugar, abordar el problema de la medición de la calidad democrática. De acuerdo con la teoría, se elabora un índice multidimensional que incluye el control del poder político, que es un elemento importante para la calidad democrática. En segundo lugar, se contrasta la capacidad explicativa de algunas de las hipótesis actuales (estructurales, socioculturales, institucionales y experiencia democrática) sobre los factores que determinan la calidad democrática. De hecho, el conocimiento de las dimensiones y de los factores que influyen en la calidad democrática tiene una justificación substantiva, ya que ello permite obtener conocimientos para consolidar la democracia (Diamond y Morlino, 2004).

El trabajo está formado por cinco secciones. En la primera, se repasa la literatura sobre la calidad democrática y sus dimensiones. En la segunda, se operacionaliza este concepto y se elabora un índice multidimensional con el que se realiza un análisis comparado en sesenta países. El tercer apartado recoge las teorías existentes sobre los factores que pueden explicar las diferencias de calidad democrática entre Estados. En la cuarta sección se plantean y contrastan seis hipótesis sobre las diferencias de calidad democrática. En la última sección se recogen las conclusiones generales.

\section{2. ¿Qué se entiende por calidad de la democracia?}

La definición de calidad de la democracia es un concepto complicado que no tiene consenso en la literatura especializada. Existen diferentes criterios e índices para estudiar y medir este concepto (Munck, 2004; Hagopian, 2005). Debido a esta falta de consenso, a veces se confunde la calidad democrática con el nivel de democratización de un régimen político. Por ejemplo, para Vargas-Culell (2011) la calidad de la democracia tiene como objetivo evaluar el estado de democratización, de acuerdo a unas determinadas características, en un punto concreto del tiempo. Pero a pesar de la diversidad de aproximaciones conceptuales, la mayoría de autores coinciden en que sólo se puede hacer análisis de calidad a los países que cumplen las condiciones esenciales de una

${ }^{1}$ Diamond (2003), definiendo la democracia como la forma de gobierno donde se escogen los gobernantes con elecciones periódicas, libres, limpias y competitivas, analiza cómo la tercera oleada de democratización se ha extendido desde el año 1974 en todo el mundo. 
democracia. Por lo tanto, no se pueden incluir los regímenes híbridos o los no democráticos en el estudio de la calidad democrática (Altman y Pérez Liñán, 2002; VargasMachuca, 2006; Morlino, 2007, 2009).

Otro problema de la calidad de la democracia es que las dimensiones que se utilizan habitualmente sobre la calidad democrática presentan problemas de inconsistencia teórica con las definiciones de democracia y con los marcos teóricos, debido a una justificación teórica débil entre el concepto de democracia y los indicadores de calidad utilizados. Los enfoques convencionales sobre la calidad democrática incluyen dimensiones con justificaciones normativas que no siempre son útiles para hacer comparaciones entre países. También las dimensiones pueden tener relaciones causales entre ellas al no ser totalmente independientes. Además, los indicadores utilizados pueden ser ambiguos y a veces están basados en percepciones subjetivas (Mazzuca, 2007; Munck, 2012).

Uno de los primeros autores que se interesan por la calidad democrática es Lijphart (1999), que concluye que las democracias consensuales gozan de una mayor calidad democrática que las mayoritarias. Aunque en los trabajos más recientes se encuentran muchas aproximaciones sobre la calidad democrática, se pueden distinguir tres enfoques.

El primer enfoque, el procedimental, parte del concepto de poliarquía ${ }^{2}$ de Dahl (1956) y se centra en evaluar los procesos e instituciones que sirven para escoger electoralmente a los gobernantes. Este enfoque analiza las reglas del juego democrático que regulan el acceso al poder político, asumiendo que la democracia es un tipo de régimen político (Altman y Pérez-Liñán, 2002; Mainwaring y Pérez-Liñán, 2008). Para este enfoque la calidad de la democracia es la capacidad de aprovechar el potencial que ofrece el régimen político democrático (Altman y Pérez-Liñán, 2002).

El segundo enfoque añade al anterior el control del poder político ya que para algunos autores esta dimensión es esencial para analizar la calidad democrática. Si bien cualquier definición de democracia, incluyendo la poliarquía, contiene el control electoral, las concepciones de democracia del primer enfoque no tienen en cuenta otras instituciones de control del poder político ni incluyen el Estado de derecho como límite a la acción de las instituciones de gobierno. O'Donnell (2004a) estima que para un buen funcionamiento democrático es necesario que el poder se ejerza bajo control. Los Estados tienen que tener un marco jurídico que garantice un sistema de derechos, con un marco legal que limite la acción de gobierno y con unas instituciones que tengan como objetivo la representación, participación y control del poder político (O’Donnell, 2004a; Vargas-Machuca, 2006, Bülmann et al., 2012).

${ }^{2}$ La poliarquía de Dahl (1956), que parte de un ideal máximo de democracia que no existe en la realidad, tiene que cumplir ocho garantías institucionales: libertad de asociación, libertad de expresión, libertad de voto, derecho al sufragio pasivo, derecho de los líderes a la competencia política, diversidad de fuentes de información, elecciones libres e imparciales e instituciones que garanticen que la política del gobierno depende de los votos. 
El control del poder político se materializa con la rendición de cuentas, que puede ser horizontal o vertical (O'Donnell, 2004a, 2004b). La primera se refiere a la existencia de instituciones estatales que tienen la autoridad y la capacidad para ejercer el control y sancionar actos u omisiones de otras instituciones del Estado (O’Donnell, 2004a). La accountability vertical se subdivide en electoral y societal. La rendición de cuentas electoral se ejerce retrospectivamente al premiar o castigar con el voto a los representantes que optan a la relección (O’Donnell, 2004b). La rendición de cuentas societal se produce en el período entre elecciones. Se trata de las acciones de un conjunto de asociaciones y movimientos con tres objetivos: hacer públicos los errores gubernamentales, introducir temas en la agenda pública y activar las agencias de accountability horizontal (Smulovitz y Peruzzotti, 2000). De hecho, la rendición de cuentas horizontal, electoral y societal están estrechamente relacionadas (O'Donnell, 2004a).

No obstante, hay autores que consideran que los mecanismos que controlan el ejercicio del poder político no se deben incluir en el análisis de la calidad democrática. Mazzuca (2007), que tiene una concepción minimalista y poliárquica de la democracia, cree que el concepto de régimen democrático se limita al acceso del poder político. En consecuencia, para evaluar la calidad de una democracia, no se deben incluir elementos relativos al ejercicio del poder político. ${ }^{3}$

El tercer enfoque amplia la concepción de democracia vista hasta ahora con contenidos sustantivos y de resultado. Además de las dimensiones relativas a los procedimientos democráticos y al control del poder político, también se incluyen objetivos y resultados de la democracia como pueden ser la igualdad socioeconómica, el nivel de bienestar o la justicia social. En la teoría política normativa se pueden encontrar numerosas concepciones de la democracia. Por ejemplo, desde una visión de la democracia deliberativa se enfatiza la importancia de la discusión pública como criterio de la calidad democrática. Habermas (1962) establece el principio de argumentación moral y de universalidad y Manin (1987) enfatiza el debate público como garantía de una buena democracia. Más actual es el artículo seminal de Diamond y Morlino (2004), que creen que la democracia tiene tres objetivos: el control de las políticas públicas, la igualdad política y el buen gobierno.

La tabla 1 recoge las dimensiones que utilizan algunos estudios para evaluar la calidad de la democracia. Se pueden observar las diferencias de criterio según el enfoque más o menos amplio de la definición de democracia.

${ }^{3}$ Algunos autores critican que los enfoques sustantivos tienen una vertiente normativa que confunde los resultados sociales y económicos de la democracia con las características definitorias del concepto calidad de la democracia (Mazzuca, 2007; Vargas-Culell, 2011). 
TABLA 1:COMPARACIÓN DE LAS DIMENSIONES DE CALIDAD DE LA DEMOCRACIA EN ALGUNOS ESTUDIOS

\begin{tabular}{|c|c|c|}
\hline \multicolumn{3}{|c|}{ ENFOQUES PROCEDIMENTALES SIN CONTROL DEL PODER POLÍTICO } \\
\hline $\begin{array}{l}\text { Mainwaring y Pérez-Liñán } \\
\qquad(2008)\end{array}$ & $\begin{array}{l}\text { Altman y Pérez-Liñán } \\
\text { (1999) }\end{array}$ & $\begin{array}{l}\text { Altman y Pérez-Liñán } \\
\text { (2002) }\end{array}$ \\
\hline $\begin{array}{l}\text { - Derechos políticos y } \\
\text { libertades civiles }\end{array}$ & $\begin{array}{l}\text { - Participación política } \\
\text { - Competencia efectiva }\end{array}$ & $\begin{array}{l}\text { - Participación política } \\
\text { - Competencia efectiva } \\
\text { - Derechos civiles }\end{array}$ \\
\hline \multicolumn{3}{|c|}{ ENFOQUES PROCEDIMENTALES CON CONTROL DEL PODER POLÍTICO } \\
\hline Vargas-Machuca (2006) & \multicolumn{2}{|c|}{ Vargas-Machuca (2013) } \\
\hline $\begin{array}{l}\text { - Estado de derecho } \\
\text { - Accountability horizontal } \\
\text { - Representatividad } \\
\text { - Oposición democrática } \\
\text { - Democracia asociativa } \\
\text { - Democracia inclusiva } \\
\text { - Sesgo deliberativo }\end{array}$ & \multicolumn{2}{|c|}{$\begin{array}{l}\text { - Estado (cohesión territorial del poder, administración } \\
\text { pública eficaz) } \\
\text { - Estado de derecho (imperio de la ley, Estado de derechos, } \\
\text { división, control y equilibrio de poderes) } \\
\text { - Representación y participación (representación } \\
\text { democrática, democracia de partidos) } \\
\text { - Sociedad (democracia asociativa, democracia y } \\
\text { oposición, competencia cívica) }\end{array}$} \\
\hline Levine y Molina (2007) & \multicolumn{2}{|c|}{ Bülmann et al. (2012) } \\
\hline $\begin{array}{l}\text { - Democracia electoral } \\
\text { - Participación } \\
\text { - Accountability } \\
\text { (horizontal, vertical, } \\
\text { societal) } \\
\text { - Respuesta a la voluntad } \\
\text { popular } \\
\text { - Soberanía (externa, } \\
\text { interna) }\end{array}$ & \multicolumn{2}{|c|}{$\begin{array}{l}\text { - Libertades individuales } \\
\text { - Estado de derecho } \\
\text { - Esfera pública } \\
\text { - Competencia } \\
\text { - Checks and balances } \\
\text { - Capacidad de gobierno } \\
\text { - Transparencia } \\
\text { - Participación } \\
\text { - Representación }\end{array}$} \\
\hline
\end{tabular}

\section{ENFOQUES SUSTANTIVOS}

Diamond y Morlino (2004) Morlino (2007)

- Estado de derecho - Estado de derecho - Legalidad

- Participación

- Competencia

- Accountability vertical - Libertades

- Accountability vertical

- Accountability horizontal

- Derechos sociales

- Accountability horizontal

- Libertad

- Libertad

- Igualdad

- Igualdad

- Responsiveness

- Calidad de las elecciones

- Transparencia

gubernamental

- Responsiveness

- Integridad

- Accesibilidad de los

ciudadanos

Fuente: Elaboración propia. Aunque Vargas-Machuca (2006), Vargas-Machuca (2013) y Bülmann et al. (2012) parten de definiciones amplias de democracia, se han incluido dentro del enfoque procedimental con control del poder político, porque utilizan dimensiones procedimentales para evaluar la calidad de la democracia. 


\section{Medir la calidad de la democracia}

Después de repasar la literatura sobre el concepto calidad de la democracia, en la segunda sección se abordará la operacionalización de este concepto. Seguidamente se realizará un análisis comparado de la calidad en sesenta democracias.

\subsection{Operacionalización del concepto}

De acuerdo con la definición de democracia electoral de Diamond (2003), se utiliza el Índice de Polity IV para seleccionar los casos. Se han escogido todos los países que desde el año 1991 y hasta 2008, de forma ininterrumpida, tienen una puntuación igual o superior a $6 .{ }^{4}$ Se trata de 60 países democráticos de todas las regiones del mundo con diversidad de características económicas, sociales, institucionales y de experiencia democrática. $^{5}$

Este trabajo, igual que la mayoría de investigaciones que analizan la calidad democrática, parte también de las definiciones procedimentales. Así, las dimensiones que se extraen de los enfoques procedimentales son tres: derechos políticos y libertades civiles, Estado de derecho y participación. A éstas se añade el control del poder político en dos vertientes: rendición de cuentas horizontal y rendición de cuentas vertical. Y también se añade la dimensión responsiveness porque conjuntamente con las dimensiones de derechos políticos y libertades civiles y participación, es un elemento clave de la poliarquía de Dahl (1956). No se puede basar el análisis de la calidad democrática en un solo indicador, como Mainwaring y Pérez-Liñán (2008), porque sería deficiente para aproximarse a un concepto poliédrico.

Se ha seleccionado un indicador cuantitativo para cada una de las seis dimensiones relativas a la calidad democrática. Los indicadores se han escogido de acuerdo con su idoneidad para medir las variables. Pero también se ha tenido en cuenta la cobertura del mayor número posible de casos y la continuidad temporal.

Para medir la dimensión derechos políticos y libertades civiles se utilizan, siguiendo la línea de otros trabajos (Levine y Molina, 2007; Barreda, 2011), los indicadores derechos políticos y libertades civiles de Freedom House (FH). Una vez hecho el promedio entre ambos indicadores, se invierte la escala original para facilitar la comparación con los otros indicadores. Así, el 1 indica la mínima garantía de derechos y libertades y el 7 la máxima.

\footnotetext{
${ }^{4}$ Por convención, todos los países que obtienen una puntuación igual o superior a 6 puntos se considera que son democráticos.

${ }^{5}$ Fiji también obtiene una puntuación igual o superior a 6 entre el período 1991 a 2008, pero no se incluye en la investigación por falta de datos de los diferentes indicadores utilizados.
} 
La dimensión Estado de derecho se analiza, igual que otros trabajos (Hagopian, 2005), con el indicador Estado de derecho del Banco Mundial (BM). Este indicador es el resultado de agregar 31 indicadores, entre los cuales se encuentran el derecho a la propiedad, la independencia judicial o la igualdad ante la ley. Sus valores oscilan entre 0 (peor puntuación) y 100 (mejor puntuación).

La participación se operacionaliza con el indicador más utilizado en los trabajos sobre la calidad democrática: la proporción de votantes sobre el total de la población en edad legal de votar. Se emplean los datos de participación en elecciones parlamentarias de International Institute for Democracy and Electoral Assistance (IDEA).

La dimensión rendición de cuentas horizontal se mide con el índice de percepción de la corrupción de Transparency International, igual que otros trabajos (Levine y Molina, 2007; Barreda, 2011). Se trata de un índice formado por la combinación de varios indicadores basados en la percepción de expertos. Los valores de este indicador varían entre 0 (alta corrupción) y 10 (alta transparencia).

El indicador que se usa para analizar la rendición de cuentas vertical es la libertad de prensa de FH, igual que Barreda $(2010,2011)$. Para facilitar el análisis, se ha invertido la escala original del indicador. Así, el 0 indica la mínima libertad de prensa y el 100 la máxima.

Por último, para medir la dimensión responsiveness, se emplea el indicador efectividad del gobierno del BM. Se trata de una variable proxy con el nivel de satisfacción de la ciudadanía con la democracia, que es el indicador más utilizado para medir esta dimensión (Hagopian, 2005; Levine y Molina, 2007; Barreda, 2011). Se opta por la efectividad del gobierno, ${ }^{6}$ porque no se encuentran datos sobre el nivel de satisfacción para el conjunto de países de este trabajo. La efectividad del gobierno es un índice agregado construido con 31 fuentes de información que avalúa la eficacia y eficiencia del gobierno, la calidad en la formulación y aplicación de las políticas públicas, la calidad y extensión de la burocracia, el malbaratamiento del gasto público y la calidad de los servicios y administraciones públicas. Los valores oscilan entre 0 (peor efectividad) y 100 (mejor efectividad).

Como se ha visto, se emplean indicadores muy diversos, En consecuencia, se encuentran algunos valores ausentes en las series de datos. Para poder realizar el análisis factorial, obteniendo un índice de calidad de la democracia y facilitar el análisis de regresión posterior, se han completado los valores ausentes con el promedio del país correspondiente.

${ }^{6}$ Para Dahl (2012) un gobierno efectivo es aquel que toma decisiones sobre los principales problemas que afectan a la ciudadanía y en los que la opinión pública cree necesaria una intervención gubernamental. 


\section{2. Índice de calidad de la democracia}

Para estudiar la calidad democrática con indicadores cuantitativos existen dos posibilidades. La primera es construir un índice agregado, como hacen Levine y Molina (2007). La segunda opción es realizar un análisis factorial de componentes principales, igual que Altman y Pérez-Liñán (2002). Pero los primeros no incluyen las dimensiones de derechos políticos y libertades civiles y Estado de derecho y los segundos no tienen en cuenta las dimensiones relativas al poder político ni la responsiveness. Esta investigación opta por el análisis factorial, ${ }^{7}$ ya que se trata de una técnica muy adecuada para estudiar un concepto formado por múltiples dimensiones y se sospecha que están relacionadas entre ellas. Además, el análisis factorial permite ver si existen una o más dimensiones latentes de los seis indicadores de la calidad democrática o bien si son independientes entre ellos.

Los resultados del análisis factorial indican que existe una dimensión latente a los seis indicadores, tal como se puede ver en la tabla 2. De hecho, este factor subyacente contribuye a explicar el $63,2 \%$ de la varianza total de las variables dependientes.

Los indicadores que están más asociados a este factor son tres. El primero es la efectividad del gobierno, que evalúa la capacidad de respuesta de las instituciones y la calidad de la formación e implementación de las políticas públicas. El segundo es el Estado de derecho, que se refiere a las instituciones que limitan el poder político y garantizan el ejercicio de los derechos políticos. Y el tercer indicador es la percepción de la corrupción, que se refiere a la rendición de cuentas horizontal, es decir, al control institucional del poder político.

TABLA 2: ANÁLISIS FACTORIAL

\begin{tabular}{lcc}
\hline Indicador & Factor 1 & Comunalidad \\
\hline Derechos políticos y libertades civiles & 0,748 & 0,559 \\
Estado de derecho & 0,946 & 0,895 \\
Participación electoral & 0,188 & 0,035 \\
Corrupción & 0,919 & 0,845 \\
Libertad de prensa & 0,733 & 0,537 \\
Efectividad & 0,959 & 0,920 \\
Variancia explicada & & $63,2 \%$ \\
\hline
\end{tabular}

Fuente: Elaboración propia.

\footnotetext{
${ }^{7}$ Análisis factorial de componentes principales con el supuesto de máxima verosimilitud y rotación varimax.
} 
Los resultados del análisis factorial confirman que en el estudio de la calidad democrática se deben incluir las dimensiones relativas al control del poder político, tal como se ha visto en los trabajos del enfoque procedimental con control del poder político (O’Donnell, 2004a, 2004b; Vargas-Machuca, 2006; Levine y Molina, 2007; Barreda, 2011; Bühlmann et al., 2012). Esto supera a los enfoques procedimentales que no tienen en cuenta los factores de control del poder político (Mainwaring y Pérez-Liñán, 2008; Altman y Pérez-Liñán, 1999, 2002). Además, estos resultados no siguen la línea argumental de Mazzuca (2007) porque confirman que en el análisis de la calidad democrática se deben incluir las dimensiones relativas al acceso al poder político y al ejercicio del poder político.

Con el factor extraído del análisis factorial se puede hacer, en primer lugar, una comparación del nivel de calidad democrática de los 60 países. Tal como se puede observar en el gráfico 1, entre los Estados con puntuaciones más altas destacan los países nórdicos, seguidos de los países de Europa Occidental y los anglosajones. Y entre los Estados con menor calidad democrática están presentes los países sud-americanos, africanos y asiáticos. Los Estados que lideran el índice de calidad democrática son Dinamarca y Finlandia, seguidos de Suecia, Noruega, Suiza y Nueva Zelanda. En cambio, destacan por la inferior calidad democrática Malasia, Zambia, Nicaragua, Islas Salomón, Honduras y Nepal. Al dividirse los 60 países en cuartiles se encuentra que, en general, los Estados del "norte" tienen una mejor calidad democrática que los Estados del "sur".

GRÁFICO1: ÍNDICE DE CALIDAD DEMOCRÁTICA POR PAÍSES.

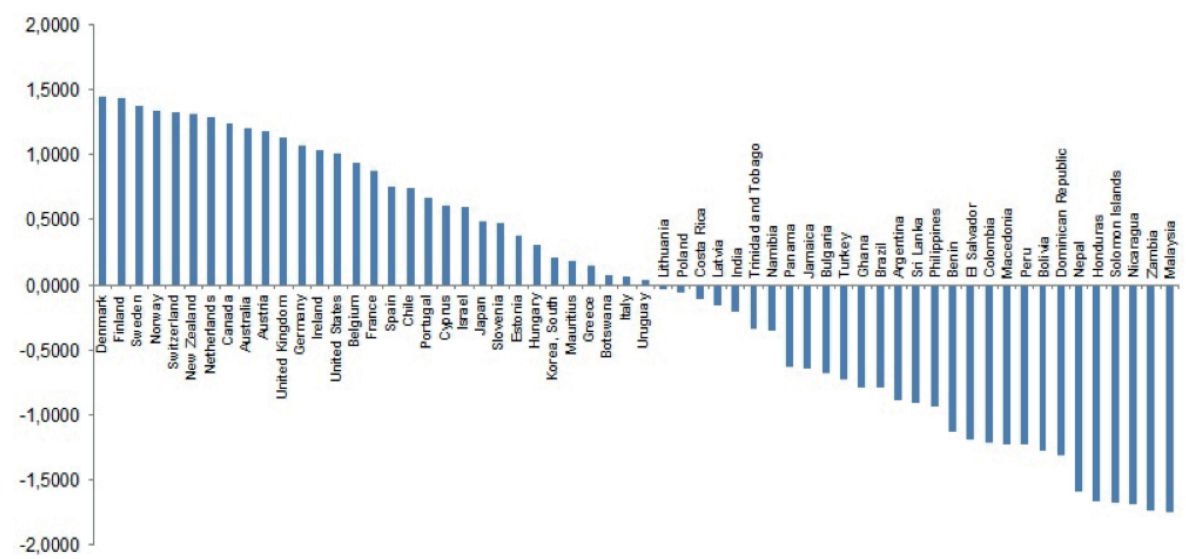

Fuente: Elaboración propia. Los valores se corresponden al promedio de 1991-2008 del análisis factorial. 
En segundo lugar se examina si existen diferencias a lo largo del tiempo. La tabla 3 muestra la evolución de la media del índice de calidad de la democracia entre tres períodos (1991-1996, 1997-2002 y 2003-2008) por cada uno de los Estados. En la mayoría de los países la calidad democrática se mantiene estable a lo largo del tiempo, con variaciones pequeñas.

Tabla 3: Media del índice de CaLidad de la democracia por países

\begin{tabular}{lcccc}
\hline & 1191 & 1997 & 2003 & 1991 \\
Estado & - & - & - & - \\
& 1996 & 2002 & 2008 & 2008 \\
\hline Denmark & 1,433 & 1,462 & 1,452 & 1,449 \\
Finland & 1,394 & 1,455 & 1,440 & 1,430 \\
Sweden & 1,363 & 1,375 & 1,376 & 1,372 \\
Norway & 1,347 & 1,335 & 1,329 & 1,337 \\
Switzerland & 1,320 & 1,327 & 1,322 & 1,323 \\
New Zealand & 1,333 & 1,290 & 1,309 & 1,310 \\
Netherlands & 1,284 & 1,306 & 1,265 & 1,285 \\
Canada & 1,247 & 1,268 & 1,221 & 1,246 \\
Australia & 1,214 & 1,193 & 1,216 & 1,208 \\
Austria & 1,172 & 1,170 & 1,207 & 1,183 \\
United Kingdom & 1,120 & 1,136 & 1,135 & 1,131 \\
Germany & 1,071 & 1,066 & 1,068 & 1,068 \\
Ireland & 1,050 & 1,015 & 1,024 & 1,030 \\
United States & 1,017 & 1,026 & 0,975 & 1,006 \\
Belgium & 0,954 & 0,859 & 1,005 & 0,940 \\
France & 0,856 & 0,828 & 0,934 & 0,873 \\
Spain & 0,701 & 0,827 & 0,723 & 0,750 \\
Chile & 0,724 & 0,706 & 0,796 & 0,742 \\
Portugal & 0,166 & 0,646 & 0,674 \\
Cyprus & 0,674 & 0,701 & 0,557 & 0,613 \\
Israel & 0,601 & 0,544 & 0,602 \\
Japan & 0,649 & 0,841 & 0,486 \\
Slovenia & 0,274 & 0,531 & 0,478 \\
Estonia & 0,646 & 0,584 & 0,381 \\
Hungary & 0,716 & 0,316 & 0,301 \\
Korea, South & 0,457 & 0,335 & 0,204 \\
Mauritius & 0,282 & 0,229 & 0,190 \\
Greece & 0,332 & 0,132 & 0,146 \\
\hline
\end{tabular}


Tabla 3: Media del índice de Calidad de la democracia por países (Continuación)

\begin{tabular}{|c|c|c|c|c|}
\hline Estado & $\begin{array}{c}1191 \\
- \\
1996\end{array}$ & $\begin{array}{c}1997 \\
- \\
2002\end{array}$ & $\begin{array}{c}2003 \\
- \\
2008\end{array}$ & $\begin{array}{c}1991 \\
- \\
2008\end{array}$ \\
\hline Botswana & 0,066 & 0,088 & 0,064 & 0,077 \\
\hline Italy & 0,059 & 0,161 & $-0,019$ & 0,067 \\
\hline Uruguay & 0,009 & $-0,016$ & 0,113 & 0,035 \\
\hline Lithuania & $-0,095$ & $-0,111$ & 0,110 & $-0,032$ \\
\hline Poland & $-0,049$ & 0,012 & $-0,140$ & $-0,059$ \\
\hline Costa Rica & $-0,113$ & $-0,054$ & $-0,154$ & $-0,107$ \\
\hline Latvia & $-0,250$ & $-0,258$ & 0,045 & $-0,154$ \\
\hline India & $-0,275$ & $-0,224$ & $-0,128$ & $-0,209$ \\
\hline Trinidad and Tobago & $-0,312$ & $-0,247$ & $-0,465$ & $-0,341$ \\
\hline Namibia & $-0,315$ & $-0,313$ & $-0,408$ & $-0,345$ \\
\hline Panama & $-0,667$ & $-0,580$ & $-0,635$ & $-0,627$ \\
\hline Jamaica & $-0,656$ & $-0,642$ & $-0,641$ & $-0,646$ \\
\hline Bulgaria & $-0,769$ & $-0,726$ & $-0,553$ & $-0,683$ \\
\hline Turkey & $-0,776$ & $-0,848$ & $-0,572$ & $-0,732$ \\
\hline Ghana & $-0,937$ & $-0,806$ & $-0,610$ & $-0,784$ \\
\hline Brazil & $-0,823$ & $-0,765$ & $-0,783$ & $-0,790$ \\
\hline Argentina & $-0,778$ & $-0,853$ & $-1,011$ & $-0,881$ \\
\hline Sri Lanka & $-0,918$ & $-0,922$ & $-0,880$ & $-0,907$ \\
\hline Philippines & $-0,959$ & $-0,845$ & $-0,998$ & $-0,934$ \\
\hline Benin & $-1,122$ & $-1,052$ & $-1,212$ & $-1,129$ \\
\hline El Salvador & $-1,280$ & $-1,265$ & $-1,023$ & $-1,189$ \\
\hline Colombia & $-1,212$ & $-1,358$ & $-1,051$ & $-1,207$ \\
\hline Macedonia & $-1,248$ & $-1,363$ & $-1,046$ & $-1,219$ \\
\hline Peru & $-1,265$ & $-1,106$ & $-1,288$ & $-1,220$ \\
\hline Bolivia & $-1,191$ & $-1,115$ & $-1,512$ & $-1,273$ \\
\hline Dominican Republic & $-1,309$ & $-1,259$ & $-1,345$ & $-1,304$ \\
\hline Nepal & $-1,482$ & $-1,505$ & $-1,789$ & $-1,592$ \\
\hline Honduras & $-1,680$ & $-1,664$ & $-1,643$ & $-1,663$ \\
\hline Solomon Islands & $-1,501$ & $-1,663$ & $-1,853$ & $-1,672$ \\
\hline Nicaragua & $-1,729$ & $-1,620$ & $-1,715$ & $-1,688$ \\
\hline Zambia & $-1,728$ & $-1,754$ & $-1,714$ & $-1,732$ \\
\hline Malaysia & $-0,098$ & $-0,170$ & $-0,023$ & $-1,746$ \\
\hline
\end{tabular}

Fuente: Elaboración propia. 
Para comprobar la consistencia y fortaleza del índice obtenido en este trabajo se ha hecho un análisis de correlación lineal de Pearson con el índice de calidad democrática de Democracy Barometer. Se trata de un índice formado por múltiples dimensiones que también parte de un concepto no minimalista de la democracia, incluyendo el control del poder político. Los dos índices coinciden en el período 1991-2001 en 25 Estados. El coeficiente de correlación entre ambos índices es de 0,639 , es decir, existe una correlación positiva fuerte entre ambos índices. Entre el índice de Barreda (2011) y el de este trabajo coinciden cinco de los seis indicadores y 13 países. La correlación entre ambos es muy fuerte, con un coeficiente de 0,899 .

\section{Factores explicativos de la calidad de la democracia}

En la sección anterior se ha visto que existen diferencias entre países respecto a la calidad democrática. En la tercera sección, se recogerá la literatura especializada sobre los factores que pueden explicar estas diferencias. No se hace un análisis exhaustivo de toda la literatura, ya que a continuación se presentan las explicaciones más destacadas. Estas explicaciones se agrupan en cuatro tipos: factores estructurales, socioculturales, institucionales y experiencia democrática.

Dentro de las explicaciones estructurales, destacan tres factores. Primero, algunos estudios relacionan el crecimiento económico con una mejor calidad de la democracia. Ciertos autores concluyen que el nivel de desarrollo económico de un país tiene una influencia, aunque moderada, en la calidad democrática (Mainwaring y Pérez-Liñán, 2008; Barreda, 2011). En este sentido, las crisis económicas afectan negativamente a la calidad democrática (Bülmann et al., 2012). Además, la estructura económica de los Estados también puede influenciar en la calidad democrática. Variables como la inflación y la distribución del mercado laboral tienen impacto en la calidad de la democracia (Mainwaring y Pérez-Liñán, 2008). Sin embargo, Mora (2013) mantiene, sin comprobarlo empíricamente, que el desarrollo económico no es determinante para la calidad democrática.

El segundo factor estructural es la desigualdad económica, que algunos trabajos asocian con una menor participación electoral y con un menor compromiso político (Crespo y Martínez, 2005). También se relaciona la desigualdad económica con la polarización política, que dificulta la confianza política. Si las desigualdades en la distribución de los recursos se producen entre grupos sociales diferentes, la desigualdad económica también se asocia con la movilización étnica e incluso con conflictos violentos. Además, la desigualdad económica también se relaciona con el debilitamiento del imperio de la ley, hecho que comporta una menor accountability (Bermeo, 2009). Otro argumento es que una elevada desigualdad económica tiene como consecuencia que las elites favorecidas no tengan incentivos para aceptar una profundización en la 
democracia ya que tienen temor a perder sus privilegios ${ }^{8}$ (Crespo y Martínez, 2005). Por ejemplo, Hagopian (2005) encuentra que las desigualdades en Brasil han impedido garantizar los derechos de ciudadanía y el Estado de derecho.

En tercer lugar, otras explicaciones estructurales se basan en el fraccionamiento etnolingüístico. Una menor fragmentación etnolingüística está asociada con una mayor calidad de la democracia (La Porta et al., 1999; Mainwaring y Pérez-Liñán, 2008). La fragmentación se relaciona con un mayor intervencionismo en la economía, menor eficiencia gubernamental, menos provisión de bienes públicos, una administración pública más pequeña y menos libertad política (La Porta et al., 1999). En cambio, algunos autores sostienen que este argumento es discutible (Mora, 2013). De hecho, Barreda (2011) concluye que el fraccionamiento etnolingüístico no influye en la calidad democrática.

Las hipótesis de los factores socioculturales establecen que el capital social es un factor explicativo de la calidad democrática. La existencia de un comportamiento cívico, con una sociedad civil activa que participa en los asuntos públicos influye positivamente en la calidad democrática (Putnam, 1993; Lipset, 1994; Morlino, 2008). Para Boix y Posner (2000) el capital social fortalece la responsabilidad democrática porque permite a la ciudadanía informarse y debatir sobre asuntos colectivos. Más recientemente, Tabellini et al. (2013) confirman empíricamente que el capital social influye en la conducta política de los parlamentarios. El efecto del capital social sobre la conducta se produce a través de la accountability. En los distritos electorales con más capital social se producen niveles más altos de rendición de cuentas del electorado. En consecuencia, los representantes de estas circunscripciones, si quieren volver a ser elegidos, tienen incentivos para tener un buen comportamiento político (no abstenerse en las votaciones parlamentarias y no ser investigados por corrupción). Sin embargo, algunos análisis encuentran que el capital social no es un factor explicativo de la calidad democrática (Barreda, 2011) a pesar de que la confianza interpersonal favorece la responsiveness (Barreda, 2013).

Entre los factores institucionales destacan tres explicaciones. La primera es que las normas electorales pueden influenciar en la calidad democrática. Persson, Tabellini y Trebbi (2001) encuentran que los sistemas electorales mayoritarios, que acostumbran a tener listas abiertas, tienden a tener menos corrupción. En cambio, los sistemas proporcionales, que suelen tener listas cerradas, tienden a tener más corrupción. Además, los países que combinan las listas cerradas y circunscripciones pequeñas son los que tienden a tener más corrupción. Prats (2008) también coincide en que los sistemas mayoritarios reducen la corrupción, aunque ligeramente.

${ }^{8}$ La hipótesis de Crespo y Martínez (2005) es que las políticas económicas basadas en el consenso de Washington han tenido como consecuencia, en los países de América Latina, una menor igualdad. A la vez, esto dificulta la consolidación de la democracia. 
El segundo factor institucional es el sistema de partidos. A excepción de Mainwaring y Pérez-Liñán (2008), la literatura no ofrece muchos estudios sobre la influencia del sistema de partidos en la calidad democrática. Desde la teoría de la gobernabilidad, para Payne (2006), en los países con más fragmentación de partidos políticos es más difícil que los partidos se pongan de acuerdo para formar coaliciones de gobierno y mantenerlas. Por lo tanto, se asocia una mayor fragmentación con una mayor probabilidad de parálisis gubernamental. Por el contrario, en los países con pocos partidos es más fácil la formación de mayorías que den apoyo al gobierno. Estos Estados tienen una gobernabilidad más estable. En cambio, Shugart (2001) argumenta que cuantos más partidos con capacidad de veto (veto players) ${ }^{9}$ haya en el parlamento, más control puede ejercer el legislativo al gobierno. Por lo tanto, un número efectivo de partidos políticos más elevado comporta una mayor accountability sobre el ejecutivo. En la literatura sobre la calidad democrática, Mainwaring y Pérez-Liñán (2008) encuentran que el multipartidismo tienen efectos negativos en la calidad democrática, excepto en el caso de las democracias presidenciales con más de 84 años, donde el multipartidismo afecta positivamente a la calidad democrática.

La tercera explicación es la institucionalización del sistema de partidos. Algunos autores (Lipset, 1994; Mainwaring y Torcal, 2005) argumentan que un sistema de partidos políticos fuertes y con un grado elevado de institucionalización aumenta la calidad democrática. Esta hipótesis es confirmada por Barreda (2011), ya que encuentra que la institucionalización fuerte del sistema de partidos se asocia con una mejor democracia. Además, una mayor volatilidad electoral influye negativamente en la garantía de derechos democráticos (Barreda, 2013).

La cuarta y última explicación de la calidad democrática se basa en la experiencia democrática de los Estados. Esta hipótesis argumenta que los países tienen un proceso de aprendizaje democrático que aumenta con el tiempo. Hay estudios que indican que una mayor experiencia democrática tiene como consecuencia una mejor calidad democrática (Altman y Pérez-Liñán, 2002). Es más, algunas investigaciones concluyen que la experiencia democrática es el factor más influyente en la calidad democrática (Mainwaring y Pérez-Liñán, 2008; Barreda, 2011). En cambio, para otros autores es el momento de nacimiento de una democracia el factor más determinante para explicar la institucionalización de los partidos. ${ }^{10}$ Las primeras democracias tienen una volatilidad electoral inferior porque se crearon identidades fuertes con una alta fidelización de los votantes hacia el partido (Mainwaring y Zoco, 2007).

${ }^{9}$ El poder de veto de un actor es la capacidad que tiene para bloquear una decisión o pedir concesiones a cambio de su consentimiento.

${ }^{10}$ Una débil institucionalización del sistema de partidos favorece, con el clientelismo, la accountability particularista en detrimento de la accountability vertical (Máiz, 2003) 


\section{Exploración de los factores explicativos de la calidad de la democracia}

Después de revisar los trabajos previos sobre los factores que pueden influir en la calidad democrática, en la cuarta sección se contrastarán seis hipótesis y se presentarán luego los resultados.

\subsection{Operacionalización de las variables}

La literatura no ofrece muchas investigaciones que analicen empíricamente qué factores explican la calidad democrática. Los trabajos de Mainwaring y Pérez-Liñán (2008) y Barreda $(2011,2013)$ tienen en cuenta diferentes variables independientes, pero se limitan a los países de América Latina. Además, Mainwaring y Pérez-Liñán (2008) sólo usan como variable dependiente el nivel de democracia de FH. Esto no tiene en cuenta algunas dimensiones esenciales para la calidad democrática.

Se han seleccionado seis hipótesis de trabajo. Del enfoque estructural se contrasta si el nivel de desarrollo económico afecta positivamente a la calidad democrática. De la perspectiva sociocultural se comprueba si el capital social influye positivamente en la calidad democrática. De las explicaciones institucionales se analiza si los sistemas electorales mayoritarios tienen mejor calidad democrática que los proporcionales. Y también se contrasta si el multipartidismo tiene efectos negativos en la calidad democrática. La última hipótesis que se comprueba es si la experiencia democrática tiene efectos positivos en la calidad democrática.

Así pues, de acuerdo con las explicaciones teóricas, se han seleccionado seis variables independientes. Para cada una de ellas se ha escogido un indicador cuantitativo de acuerdo a su idoneidad, cobertura del mayor número de casos y continuidad temporal.

De las explicaciones estructurales se estudian dos variables: el nivel de desarrollo y la desigualdad económica. La primera se operacionaliza con el PIB per cápita del BM. La segunda se mide con el Índice de Gini del BM, donde el valor 0 indica una equidad perfecta y el 100 una desigualdad absoluta.

Entre las explicaciones socioculturales se analiza la variable capital social, que se operacionaliza con el indicador confianza interpersonal del World Values Survey, recopilado en el The Quality of Government Dataset (QOG). Se trata de un índice que recoge de diversas encuestas internacionales la pregunta sobre el grado de confianza hacia las otras personas. Los valores oscilan entre 0 (necesidad de tener cuidado con los otros ciudadanos) y el 1 (se puede confiar en mucha gente).

De las explicaciones institucionales se consideran las variables sistema electoral y sistema de partidos. La primera se analiza con el indicador sistema electoral del QOG y los datos no disponibles se completan con información de IDEA. Se ha transformado esta variable en una variable dummy. El valor 0 indica un sistema electoral mayoritario y el valor 1 un sistema proporcional o con algún elemento de proporcionalidad. 
El sistema de partidos se operacionaliza con el indicador número efectivo de partidos electorales del QOG. Este indicador mide el número efectivo de partidos que compiten electoralmente basado en la fórmula de Laakso y Taagepera (1979). Los valores más altos indican un mayor fraccionamiento.

Finalmente, la variable experiencia democrática se estudia con un indicador de elaboración propia con datos de Polity IV. Se ha empezado a contar desde el año 1946 y sumado un año de experiencia por cada año que el país obtiene una puntuación igual o superior a 6 de forma ininterrumpida.

Además, esta investigación incluye la educación como variable de control ya que afecta a la vez a las variables de la calidad democrática y a algunas variables independientes. La educación es necesaria para que la ciudadanía tenga mejor capacidad para tomar decisiones políticas. Esto favorece una mayor participación política y una rendición de cuentas vertical más efectiva. También se relacionan niveles más altos de educación con una mayor igualdad social (Diamond y Morlino, 2004; Levine y Molina, 2007). La educación también está asociada con el desarrollo económico (Barro y Lee, 2011) y con menores niveles de corrupción (Prats, 2008). Esta variable, se operacionaliza con el indicador índice de educación del PNUD, que describe el promedio de años recibidos de educación de la población de 25 años o más

\subsection{Contraste de hipótesis}

Se realiza un análisis de regresión múltiple de cuadrados mínimos para estudiar cómo afectan las seis variables independientes al índice de democracia obtenido con el análisis factorial. Además, se tiene en cuenta la educación como variable de control. Para solucionar los problemas de endogeneidad de las variables nivel de desarrollo y educación en relación a la calidad democrática, se han tratado las dos primeras como variables rezagadas. Se trata de usar los valores del PIB y del índice de educación del año anterior respecto al índice de calidad. Por ejemplo, para el año 1991 se usa el valor del PIB del año 1990; para el año 1992 el PIB de 1991, y así sucesivamente. Se han completado los valores vacíos de los indicadores confianza interpersonal e índice de educación con el promedio del país correspondiente.

El modelo que se presenta explica el 67,3\% de la variabilidad del índice de calidad democrática y es significativo con un nivel de confianza del 99\%. El resultado de la regresión, tal como se puede ver en la tabla 4, indica que las variables estructurales no son significativas para explicar las variaciones de la calidad democrática. El PIB, que mide el desarrollo económico, no es significativo. Esto apoya a Mora (2013), que hipotetiza, sin comprobarlo empíricamente, que el desarrollo económico no es determinante para la calidad de la democracia. Así pues, este resultado no se ajusta a los trabajos de Mainwaring y Pérez-Liñán (2008) y Barreda (2011), que concluyen que el desarrollo económico tiene un efecto positivamente moderado en la calidad democrática. El índice de 
Gini, que mide la desigualdad, tampoco es significativo. Esto contradice a la literatura que postula que la desigualdad social influye negativamente en la calidad democrática (Bermeo, 2009; Crespo y Martínez, 2005; Barreda, 2011).

TABLA 4: ANÁLISIS DE REGRESIÓN

\begin{tabular}{lcc} 
& \multicolumn{1}{c}{$\beta^{\wedge}$} & $\mathrm{t}$ \\
\hline PIB & 0,00000000 & 0,24 \\
Índice de Gini & $-0,001069$ & $-0,22$ \\
Confianza interpersonal & $2,5537^{*}$ & 5,56 \\
Sistema proporcional & $-0,3853^{*}$ & $-2,72$ \\
Número efectivo de partidos & $0,10845^{*}$ & 6,70 \\
Experiencia democrática & $0,008784^{*}$ & 3,04 \\
Índice de educación & $0,17424^{*}$ & 6,67 \\
Constante & $-2,3263$ & $-5,18$ \\
$\mathrm{R}^{2}$ corregida & & 0,669 \\
Observaciones & \multicolumn{2}{c}{} \\
\hline
\end{tabular}

Fuente: Elaboración propia. Análisis de regresión múltiple de cuadrados mínimos, * indica un nivel de significación del 99\%.

La regresión confirma la hipótesis de que los sistemas mayoritarios tienen una mejor calidad democrática que los proporcionales. Los resultados indican que los sistemas mayoritarios tienden a tener una puntuación más elevada en la calidad democrática que los proporcionales (coeficiente Beta de -0,3691). Esto se opone a Lijphart (1999) que obtuvo que las democracias consensuales son de mejor calidad que las mayoritarias. En cambio, la regresión da un resultado opuesto a lo que se esperaba en la otra hipótesis institucional. El coeficiente Beta de 0,10845 indica que los países con un número efectivo de partidos mayor tienden a tener un poco mejor las puntuaciones en calidad democrática. Por lo tanto, no se confirma la hipótesis de que la fragmentación del sistema de partidos influye negativamente en la calidad democrática, en oposición a Maiwaring y Pérez-Liñán (2008). En cambio, este resultado ratifica que la fragmentación del sistema de partidos influye positivamente a la calidad democrática, ya que un número más elevado de partidos con capacidad de veto en el parlamento aumenta la capacidad de accountability del poder legislativo sobre el ejecutivo Shugart (2001).

El modelo muestra que el capital social tiene efectos positivos sobre la calidad de la democracia y, además, es la variable más determinante. El indicador confianza interpersonal tiene un coeficiente Beta de 2,5537. Esto sitúa este trabajo en la misma línea que ostros estudios sobre la explicación sociocultural (Putnam, 1993; Lipset, 1994; Boix y Posner, 2000; Morlino, 2008; Tabellini et al., 2013). 
Finalmente, igual que otros trabajos anteriores (Altman y Pérez-Liñán, 2002; Mainwaring y Pérez-Liñán, 2008; Barreda, 2011), la regresión confirma que la experiencia democrática influye positivamente en la calidad democrática, aunque moderadamente (coeficiente Beta de 0,008784).

\section{Conclusiones}

En la literatura sobre la calidad de la democracia se hallan trabajos que tienen una concepción mínima del concepto democracia y trabajos que tienen una concepción más amplia de este concepto. Esta investigación parte de una concepción no minimalista de la democracia, que incluye la vertiente del control al poder político. En la primera parte de este trabajo se seleccionan seis dimensiones (derechos políticos y libertades civiles, Estado de derecho, participación, rendición de cuentas horizontal, rendición de cuentas vertical y responsiveness) para construir un índice multidimensional de calidad democrática y aplicarlo sesenta países. A partir de este índice, se pueden destacar varias observaciones.

El análisis factorial confirma que es necesario incluir en el análisis de la calidad de la democracia las dimensiones relativas al control del poder político, siguiendo la línea de los trabajos de O'Donnell (2004a, 2004b), Vargas-Machuca (2006), Levine y Molina, (2007), Barreda (2011) y Bühlmann et al., (2012). Por lo tanto, no se tiene que limitar el estudio de la calidad de la democracia con sólo las dimensiones relacionadas con los derechos democráticos, superando a los enfoques que no tienen en cuenta el control del poder político (Mainwaring y Pérez-Liñán, 2008; Altman y Pérez-Liñán, 1999, 2002) o rechazando las concepciones de democracia que sólo tienen en cuenta el acceso al poder político Mazzuca (2007). Se ha comprobado que existe un factor subyacente detrás de las seis dimensiones incluidas en el análisis factorial. De estas seis dimensiones, las que más se asocian al factor subyacente (de más a menos) son la responsiveness, el Estado de derecho y la rendición de cuentas horizontal.

También se observa que, si se comparan los 60 países de este trabajo, existen diferencias de calidad de la democracia entre Estados. Los países con una puntuación más elevada en el índice de calidad de la democracia son Suecia, Noruega, Suiza y Nueva Zelanda. En cambio, los países con peor calidad democrática son Malasia, Zambia, Nicaragua, Islas Salomón, Honduras y Nepal. Además, el índice de calidad de la democracia constata una brecha en términos de calidad democrática entre los países del "norte", que tienen mejores puntuaciones, y los países del "sur", que tienen peores puntuaciones. Sin embargo, no hay diferencias significativas en calidad democrática a lo largo del tiempo, a excepción de unos pocos Estados y con pocas variaciones.

En la segunda parte de este trabajo se buscan, mediante una regresión múltiple, qué factores pueden contribuir a explicar las variaciones de calidad democrática entre 
países. Estos resultados no son definitivos ya que, aunque los indicadores utilizados son correctos para contrastar las hipótesis estudiadas, son insuficientes para descartar totalmente una teoría o afirmar que unas teorías son más válidas que otras para explicar la calidad de la democracia. Se debería construir un modelo con más indicadores para estudiar la complejidad de la calidad democrática. Sin pretensión de ser concluyentes, se pueden destacar los siguientes hallazgos.

Se ha encontrado inesperadamente que las explicaciones estructurales no son significativas para explicar la calidad democrática, apoyando la hipótesis de Mora (2013). En el análisis de regresión efectuado, el nivel de desarrollo no es significativo. Este resultado no se ajusta a los trabajos de Mainwaring y Pérez-Liñán (2008) y Barreda (2011). Tampoco es significativa la desigualdad económica, contradiciendo a Bermeo, (2009), Crespo y Martínez (2005) y Barreda (2011). De las explicaciones institucionales la regresión confirma que los sistemas electorales mayoritarios tienden a tener mejor calidad democrática que los proporcionales, contradiciendo a Lijphart (1999). En cambio, se ha encontrado inesperadamente que no se confirma que el multipartidismo influya negativamente en la calidad de la democracia. Así, en este estudio no se confirma la hipótesis de Mainwaring y Pérez-Liñán (2008) pero se ratifica que, mediante de la accountability, la fragmentación del sistema de partidos influye positivamente a la calidad democrática (Shugart, 2001).

Esta investigación, de acuerdo con Putnam (1993), Lipset (1994), Boix y Posner (2000), Morlino (2008) y Tabellini et al. (2013) confirma que el capital social tiene efectos positivos en la calidad democrática. De hecho, los resultados del análisis de regresión indican que la explicación sociocultural es la más determinante para explicar la variación en la calidad democrática. Y finalmente, también se confirma que la experiencia democrática influye positivamente en la calidad democrática, aunque de forma moderada (Altman y Pérez-Liñán, 2002; Mainwaring y Pérez-Liñán, 2008; Barreda, 2011).

$* * *$

Agradecimientos

Este paper es un resumen del Trabajo Final del Máster Análisis Político de la Universitat Oberta de Catalunya. El autor desea agradecer a Mikel Barreda la tutoría, los comentarios y la ayuda prestada en la realización del trabajo original, así como las observaciones de dos evaluadores anónimos. El autor también desea agradecer las observaciones del editor y de los dos revisores. Y también agradecer a Vinyet Quesada su ayuda y sus valiosos comentarios. 


\section{Bibliografía}

Altman, D. y A. PÉrez-Liñán (1999): “Más allá de la poliarquía: una aproximación a la calidad de las democracias”, Revista Uruguaya de Ciencia Política, 11, pp. 83-105.

Altman, D. \& A. Pérez-Liñán (2002): “Assessing the quality of democracy: freedom, competitiviness and participation in eighteen latin american countries", Democratization, 9 (2), pp. 85-100.

BARreda, M. (2010): Accountability y calidad de la democracia en América Latina: un análisis comparado. Disponible en:

$<$ http://www.regionalcentrelacundp.org/images/stories/DESCENTRALIZACION/ boletin/accountability.pdf $>$ [Consulta: 20 de junio de 2013]

Barreda, M. (2011): “La calidad de la democracia. Un análisis comparado de América Latina”, Política y Gobierno, 18 (2), pp. 265-295.

BARREDA, M. (2013): "La calidad de las democracias latinoamericanas: medición y claves explicativas”. En: Mantilla, Sebastián y Munck, Gerardo. 2013. La calidad de la democracia: perspectivas desde América Latina. Quito, Centro Latinoamericano de Estudios Políticos.

BARro, R. \& J.W. LEE (2011): A new data set of educational attainment in the world, 19502010. Disponible en:

$<$ http://www.barrolee.com/papers/Barro_Lee_Human_Capital_Update_2011Nov.pdf $>$ [Consulta: 16 de junio de 2013]

Bermeo, N. (2009): "Does electoral democracy boost economic equality?", Journal of Democracy, 14 (4), pp. 21-35.

Boix, C. y D.E. Posner (2000): “Capital social y democracia”, Revista Española de Ciencia Política, 1 (2), pp. 159-1985.

Bülmann, M. et al. (2012): "The democracy barometer: A new instrument to measure the quality of democracy and its potential for comparative research", European Political Science, 11 (4), pp. 519-536.

Crespo, I. y A. Martínez (2005): "La calidad de la democracia en América Latina", Studia Politicae, 5, pp. 5-35.

DAHL, R.(1956): A preface to democratic theory. Chicago, The University of Chicago Press.

DAHL, R. (2012): La democracia. Barcelona, Editorial Ariel.

DiAmond, L. (2003): “¿Puede el mundo entero ser democrático? Democracia, desarrollo y factores internacionales", Revista Española de Ciencia Política, 9, pp. 9-38.

Diamond, L. \& L. Morlino (2004): “The quality of democracy. An overview", Journal of Democracy, 15 (4), pp. 20-31.

Escobar, M. (2011): “La calidad democrárica. Una propuesta para su medición por expertos”, Revista Española de Investigaciones Sociológicas, 133, pp. 59-80. 
Habermas, J. (1962): Escritos políticos. Madrid, Tecnos.

HaGopian, F. (2005): "Derechos, representación y la creciente calidad de la democracia en Brasil y Chile", Política y Gobierno, 12 (1), pp. 41-90.

LAAKso, M. \& R. TAagePera (1979): "The effective number of parties: a measure with application to Western Europe", Comparative Political Studies, 12, pp. 3-27.

Levine, D.H. y J.E. Molina (2007): "La calidad de la democracia en América Latina: una visión comparada", América Latina Hoy, 45, pp. 17-46.

LijPHART, A. (1999): Patterns of democracy: government forms and performance in thirtysix countries. New Haven, Yale University Press.

LiPSET, S.M. (1994): "The social requisits of democracy revisited", American Sociological Review, 59, pp. 1-22.

MAINWARING, S. \& A. Pérez-LiÑÁn (2008): Regime legacies and democratization: explaining variance in the level of democracy in Latin America, 1978-2004. Disponible en:

$<$ http://kellogg.nd.edu/publications/workingpapers/WPS/354.pdf $>$ [Consulta: 13 de abril de 2013]

MaInwaring, S. y M. Torcal (2005): "La institucionalización de los sistemas de partidos y la teoría del sistema partidista después de la tercera ola democratizadora", América Latina Hoy, 41, pp. 141-173.

Mainwaring, S. y E. Zoco (2007): "Secuencias políticas y estabilización de la competencia partidista: volatilidad electoral en viejas y nuevas democracias", América Latina Hoy, 46, pp. 147-171.

Máız, R. (2003): "La corrupción como mecanismo de autorrefurzo del clientelismo político", Revista Mexicana de Sociología, 1, pp. 3-39.

Manin, B. (1987): "On legitimacy and political deliberation”, Political Theory, 15, pp. 338-368.

MAZZUCA, S.L. (2007): "Reconceptualizing democratization: acces to power versus exercise of power". En: Munck, Gerardo. 2007. Regimes and Democracy in Laitin America: Theories and Methods. New York, Oxford University Press.

Mora, J.J. (2013): Calidad y democracia. Del sistema electoral a la rendición de cuentas. Madrid, Editorial Tecnos.

Morlino, L. (2007): "Explicar la calidad de la democracia: ¿Qué tan relevantes son las tradiciones autoritarias?", Revista de Ciencia Política, 27 (2), pp. 3-22.

MorLINo, L. (2008): Calidad democrática entre líderes y partidos. Disponible en: $<$ http://www.ife.org.mx/docs/IFEv2/CDD/CDDEventosForosAcademicos/ EventosForos-2008/PPs-CalidadDemocracia/PPs-CalidadDemocracia-docs/ Conferencia01-LeonardoMorlino.pdf> [Consulta: 07 de abril de 2013]

MorLino, L.(2009): "La calidad de la democracia”, Claves de Razón Práctica, 193, pp. 26-35. 
Munck, G. (2004): “La política democrática en América Latina: contribuciones de una perspectiva institucional” Politica y Gobierno, 25 (2), pp. 315-346.

Munck, G. (2012): Conceptualizing the quality of democracy. The framing of a new agenda for comparative politics. Disponible en:

$<$ http://kellogg.nd.edu/odonnell/papers/munck.pdf $>$ [Consulta: 03 de juny de 2013]

O’Donnell, G.A. (2004a): “Accountability horizontal: la institucionalización de la desconfianza política”, Revista Española de Ciencia Política, 11, pp. 11-31.

O’Donnell, G.A. (2004b): "Why the rule of law matters", Journal of Democracy, 15 (4), pp. 32-46.

PAYne, M. (2006): Sistemas de partidos y gobernabilidad democrática. Disponible en:

$<$ http://idbdocs.iadb.org/wsdocs/getdocument.aspx?docnum=419931 $>$ [Consulta: 06 de diciembre de 2013]

Persson, T.; G. TABellini \& F. Trebbi ( ): Electoral rules and corruption. Disponible en: $<$ http://www.cesifo-group.de/portal/page/portal/DocBase_Content/WP/WPCESifo Working_Papers/wp-cesifo-2001/wp-cesifo-2001-01/cesifo_wp416.pdf> [Consulta: 11 de junio de 2013]

Prats, J.O. (2008): “Causas políticas y consecuencias sociales de la corrupción” Papers, 88 , pp. 153-164.

Putnam, R.D. (1993): Making democracy work. Civic traditions in modern Italy. Princeton, Princeton University Press.

Shugart, M.S. (2001): "Sistemas de gobierno en América Latina: frenos y contrapesos en una era de la globalización" En: Carrillo, Fernando. 2001. Democracia en déficit. Gobernabilidad y desarrollo en América Latina y el Caribe. México, Banco Interamericano de Desarrollo.

Smulovitz, C. \& E. Pruzzotti (2000): "Societal accountability in Latin America”, Journal of Democracy, 11 (4), pp. 147-158.

TABELlini, G. et al. (2013): "Social capital and political accountability", Americal Economic Journal: Economy Policy, 5 (2), pp. 222-250.

VArgas-Culell, J. (2011): "La calidad de la democracia y el estudio comparado de la democratización”, Revista Latinoamericana de Política Comparada, 5, pp. 67-94.

Vargas-Machuca, R. (2006): La calidad de la democracia”, Claves de Razón Práctica, 165, pp. 34-41.

VARGAS-MachucA, R. (2013): "Cuando no bastan los principios: la democracia como modelo" En: Robles, Antonio y Vargas-Machuca, Ramón Arturo. 2013. La buena democracia. Claves de su calidad. Granada, Editorial Universidad de Granada. 


\section{Bases de datos}

Democracy Barometer [en línea]: Democracy Barometer Project. [Consulta: 2 de novimbre de 2013] Disponible en:

$<\mathrm{http}: / /$ www.democracybarometer.org/>

Freedom House [en línea]: Freedom in the World. [Consulta: 30 de julio de 2013] Disponible en:

$<$ http://www.freedomhouse.org $>$

International Institute for Democracy and Electoral Assistance [en línea]: Voter Turnout Database. [Consulta: 19 de junio de 2013] Disponible en:

$<$ http://www.idea.int/vt/viewdata.cfm>

Polity IV Project [en línea]: Political Regime Characteristics and Transitions, 1800-2011.

[Consulta: 20 de marzo de 2013] Disponible en:

$<$ http://www.systemicpeace.org/polity/polity4.htm>

Programa de las Naciones Unidas para el Desarrollo (PNUD) [en línea]: Indicadores Internacionales sobre Desarrollo Humano. [Consulta: 16 de junio de 2013] Disponible en:

$<$ http://hdrstats.undp.org/es/indicadores/default.html $>$

The Quality Institute of Government [en línea]: QOG Institute's data pages. [Consulta: 19 de junio de 2013] Disponible en:

$<$ http://www.qog.pol.gu.se/data/>

Transparency International [en línea]: The Corruption Perceptions Index. [Consulta: 20 de junio de 2013] Disponible en:

$<$ http://cpi.transparency.org/cpi2012/in_detail/>

World BANK [en línea]: Databank. [Consulta: 16 de junio de 2013] Disponible en:

$<\mathrm{http} / / /$ datos.bancomundial.org/>

World Bank [en línea]: The Worldwide Governance Indicators (WGI) Project. [Consulta: 26 de agosto de 2013] Disponible en:

$<$ http://info.worldbank.org/governance/wgi/index.asp $>$

World Values Survey [en línea]: World Values Suervey 1981-2008 Official Agregate v.20090901, 2009. [Consulta: 15 de julio de 2013] Disponible en:

$<$ http://www.wvsevsdb.com/wvs/WVSData.jsp $>$ 


\section{Anexos}

Países de la investigación

Argentina, Australia, Austria, Belgium, Benin, Bolivia, Botswana, Brazil, Bulgaria, Canada, Chile, Colombia, Costa Rica, Cyprus, Denmark, DominicanRepublic, El Salvador, Estonia, Finland, France, Germany, Ghana, Greece, Honduras, Hungary, India, Ireland, Israel, Italy, Jamaica, Japan, Korea, South, Latvia, Lithuania, Macedonia, Malaysia, Mauritius, Namibia, Nepal, Netherlands, New Zealand, Nicaragua, Norway, Panama, Peru, Philippines, Poland, Portugal, Slovenia, SolomonIslands, Spain, Sri Lanka, Sweden, Switzerland, Trinidad and Tobago, Turkey, United Kingdom, United States, Uruguay, Zambia.

TABLA 5: RELACIÓN DE INDICADORES UTILIZADOS DE LAS VARIABLES DEPENDIENTES

\begin{tabular}{|c|c|c|}
\hline Variable dependiente & Indicador & Fuente \\
\hline $\begin{array}{l}\text { Derechos políticos y } \\
\text { libertades civiles }\end{array}$ & $\begin{array}{l}\text { Media de derechos } \\
\text { políticos y libertades } \\
\text { civiles (1991-2008) }\end{array}$ & Freedom House \\
\hline Estado de derecho & $\begin{array}{l}\text { Estado de derecho } \\
(1991-2008)\end{array}$ & World Bank \\
\hline Participación & $\begin{array}{l}\text { Participación electoral } \\
(1991-2008)\end{array}$ & $\begin{array}{l}\text { International Institute for } \\
\text { Democracy and Electoral } \\
\text { Assistance }\end{array}$ \\
\hline $\begin{array}{l}\text { Rendición de cuentas } \\
\text { horizontal }\end{array}$ & $\begin{array}{l}\text { Índice de percepción de } \\
\text { la corrupción (1991- } \\
\text { 2008) }\end{array}$ & Transparency International \\
\hline Rendición de cuentas vertical & $\begin{array}{l}\text { Libertad de prensa } \\
(1991-2008)\end{array}$ & Freedom House \\
\hline Responsiveness & $\begin{array}{l}\text { Efectividad del } \\
\text { gobierno (1991-2008) }\end{array}$ & World Bank \\
\hline
\end{tabular}

Fuente: Elaboración propia. * Datos obtenidos en el peíodo 1990-2007. 
TABLA 6: RELACIÓN DE INDICADORES UTILIZADOS DE LAS VARIABLES INDEPENDIENTES

\begin{tabular}{|c|c|c|c|}
\hline Enfoque & $\begin{array}{c}\text { Variable } \\
\text { independiente }\end{array}$ & Indicador & Fuente \\
\hline \multirow[t]{2}{*}{ Estructural } & $\begin{array}{l}\text { Nivel de } \\
\text { desarrollo }\end{array}$ & $\begin{array}{l}\text { PIB por cápita } \\
(1991-2008)^{*}\end{array}$ & World Bank \\
\hline & $\begin{array}{l}\text { Desigualdad } \\
\text { económica }\end{array}$ & $\begin{array}{l}\text { Índice de Gini } \\
(1991-2008)\end{array}$ & World Bank \\
\hline Sociocultural & Capital social & $\begin{array}{l}\text { Confianza interpersonal } \\
(1991-2008)\end{array}$ & $\begin{array}{l}\text { World Values Survey, } \\
\text { recopilat a Quality of } \\
\text { Government Institute }\end{array}$ \\
\hline \multirow[t]{2}{*}{ Institucional } & Sistema electoral & $\begin{array}{l}\text { Sistema electoral } \\
\text { mayoritario, } \\
\text { proporcional o mixto } \\
(1991-2008)\end{array}$ & $\begin{array}{l}\text { Quality of } \\
\text { Government Institute } \\
\text { y International } \\
\text { Institute for } \\
\text { Democracy and } \\
\text { Electoral Assistance }\end{array}$ \\
\hline & $\begin{array}{l}\text { Sistema de } \\
\text { partidos }\end{array}$ & $\begin{array}{l}\text { Número efectivo de } \\
\text { partidos electorales } \\
(1991-2008)\end{array}$ & $\begin{array}{l}\text { Quality of } \\
\text { Government Institute }\end{array}$ \\
\hline $\begin{array}{l}\text { Experiencia } \\
\text { democrática }\end{array}$ & $\begin{array}{l}\text { Años de } \\
\text { experiencia } \\
\text { democrática }\end{array}$ & $\begin{array}{l}\text { Número de años } \\
\text { ininterrumpidos siendo } \\
\text { una democracia } \\
(1991-2008)\end{array}$ & $\begin{array}{l}\text { Elaboración propia } \\
\text { con datos de Polity IV }\end{array}$ \\
\hline
\end{tabular}

Fuente: Elaboración propia. * Datos obtenidos en el peíodo 1990-2007.

TABLA 7: RELACIÓN DE INDICADORES UTILIZADOS DE LA VARIABLE DE CONTROL.

\begin{tabular}{cll}
$\begin{array}{c}\text { Variable de } \\
\text { control }\end{array}$ & \multicolumn{1}{c}{ Indicador } & Fuente \\
\hline Educación & $\begin{array}{l}\text { Índice de educación } \\
(1991-2008) *\end{array}$ & $\begin{array}{l}\text { Programa de las Naciones Unidas } \\
\text { para el Desarrollo }\end{array}$ \\
\hline
\end{tabular}

Fuente: Elaboración propia. * Datos obtenidos en el período 1990-2007. 